\title{
Universiteit
}

Leiden

The Netherlands

\section{Refractive Index Matched Scanning of Dense Granular Materials}

Dijksman, J.A.; Rietz, F.; Lorincz, K.; Hecke, M.L. van; Losert, W.

\section{Citation}

Dijksman, J. A., Rietz, F., Lorincz, K., Hecke, M. L. van, \& Losert, W. (2012). Refractive Index Matched Scanning of Dense Granular Materials. Review Of Scientific Instruments, 83, 011301. doi:10.1063/1.3674173

Version: $\quad$ Not Applicable (or Unknown)

License: $\quad$ Leiden University Non-exclusive license

Downloaded from: https://hdl.handle.net/1887/61351

Note: To cite this publication please use the final published version (if applicable). 
Invited Article: Refractive index matched scanning of dense granular materials

Joshua A. Dijksman, Frank Rietz, Kinga A. Lőrincz, Martin van Hecke, and Wolfgang Losert

Citation: Review of Scientific Instruments 83, 011301 (2012); doi: 10.1063/1.3674173

View online: http://dx.doi.org/10.1063/1.3674173

View Table of Contents: http://aip.scitation.org/toc/rsi/83/1

Published by the American Institute of Physics

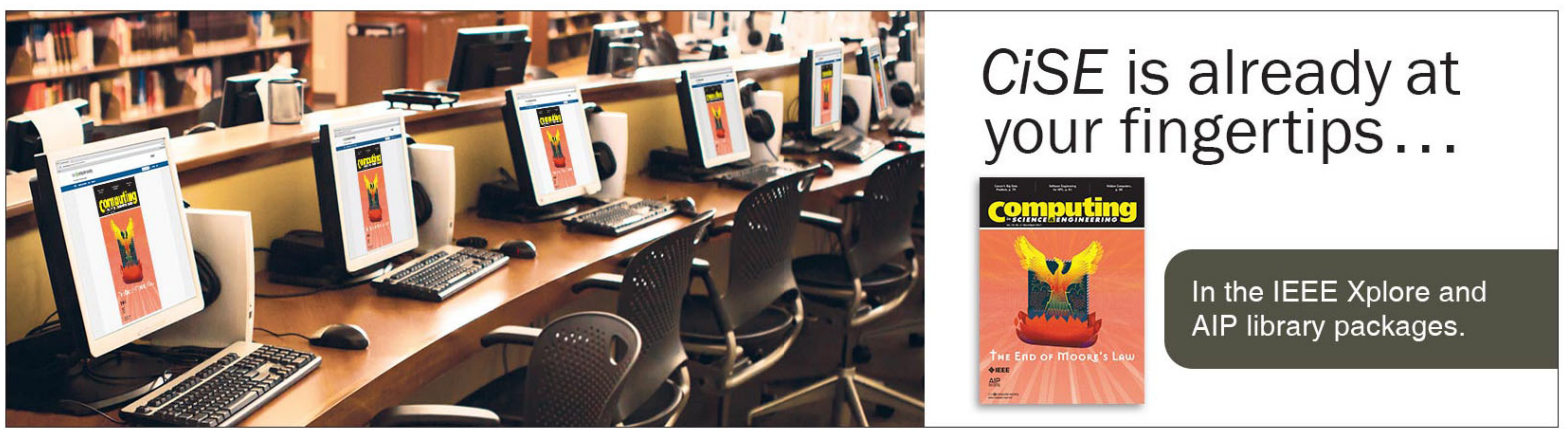




\title{
Invited Article: Refractive index matched scanning of dense granular materials
}

\author{
Joshua A. Dijksman, ${ }^{1}$ Frank Rietz, ${ }^{2}$ Kinga A. Lörincz, ${ }^{3}$ Martin van Hecke, ${ }^{4}$ \\ and Wolfgang Losert ${ }^{5}$ \\ ${ }^{1}$ Physics Department, Duke University, Box 90305, Durham, North Carolina 27708-0305, USA \\ ${ }^{2}$ Max-Planck-Institute for Dynamics and Self-Organization, Am Faßberg 17, 37077 Göttingen, \\ Germany; Department of Nonlinear Phenomena, University Magdeburg, Universitätsplatz 2, \\ 39106 Magdeburg, Germany; and Center for Nonlinear Dynamics, University of Texas at Austin, \\ 1 University Station, C1610, Austin, Texas 78712, USA \\ ${ }^{3}$ Universiteit van Amsterdam, Science Park 904, 1098 XH Amsterdam, The Netherlands \\ ${ }^{4}$ Kamerlingh Onnes Lab, Universiteit Leiden, Postbus 9504, 2300 RA Leiden, The Netherlands \\ ${ }^{5}$ Department of Physics, IPST, and IREAP, University of Maryland, College Park, Maryland 20742, USA
}

(Received 18 April 2011; accepted 1 September 2011; published online 24 January 2012)

\begin{abstract}
We review an experimental method that allows to probe the time-dependent structure of fully threedimensional densely packed granular materials and suspensions by means of particle recognition. The method relies on submersing a granular medium in a refractive index matched fluid. This makes the resulting suspension transparent. The granular medium is then visualized by exciting, layer by layer, the fluorescent dye in the fluid phase. We collect references and unreported experimental know-how to provide a solid background for future development of the technique, both for new and experienced users. ( 2012 American Institute of Physics. [doi:10.1063/1.3674173]
\end{abstract}

\section{INTRODUCTION}

Understanding the mechanical behavior of granular materials has proven to be a great challenge. Phenomena such as fracture, shear localization, and jamming ${ }^{1-5}$ are commonly observed in these materials, yet at present there is no consensus on their explanation. One major obstacle in the study of granular materials is that they are optically opaque - only their surface is directly visible.

Here, we will review an instrument that allows for the fully three-dimensional imaging and tracking of particles in a dense packing. The instrument is based on index matching: optical access to the bulk of the granular material is accomplished by immersing transparent particles in a fluid with the same index of refraction. This makes the resulting medium transparent. The bulk is then visualized by exciting, layer by layer, the fluorescent dye in the fluid phase, and capturing the resulting cross sections with a digital camera.

Refractive index matched imaging (RIM) has become a popular experimental tool in recent years, yet there are a few other experimental techniques to study individual particle dynamics of granular materials in three dimensions. Among them are X-ray tomography, magnetic resonance imaging (MRI), and confocal imaging. X-ray tomography has been used to reconstruct the three-dimensional packing structure of granular piles ${ }^{6-9}$ and has been used to investigate the overall density of vibrated granular beds. ${ }^{10} \mathrm{X}$-ray tomography can achieve very high spatial resolution. Temporal resolution is typically $\simeq$ hours per scan, but can be reduced to seconds. ${ }^{11}$ Analysis of experimental data is computationally expensive. Due to the ionizing energies of X-rays, also personal safety and sample integrity is a concern. MRI techniques have been used extensively to study flow profiles and density profiles inside sheared granulates; see for example Refs. 12-14. MRI techniques have a sub-millimeter accu- racy in macroscopic volumes, but only at a slow scanning rate. They are however limited to tracking of materials that contain hydrogen atoms,${ }^{15}$ such as organic materials. Confocal imaging can nowadays image quickly, and with high resolution, both simple cross sections and complete volumes. It can only be used for index matched ${ }^{16}$ suspensions, since its imaging technique uses visible light to access the interior. It has, however, been developed to study small systems, with particle sizes ranging from tens of nanometers to a few tens of micrometers, ${ }^{17}$ and scaling this technique up to systems with millimeter-sized particles poses significant challenges. ${ }^{18}$

We shall discuss here a particular version of RIM that works as a tomographic technique: a laser sheet is used to highlight a slice of the fluorescently labelled and refractive index matched fluid phase in the suspension. A stack of slices can then be imaged and digital imaging techniques are then used to obtain three-dimensional data from this stack. We dub this technique refractive index matched scanning (RIMS). The advantages of RIMS over the other three-dimensional scanning methods mentioned above can now be summarized: the single slice illumination technique allows one to image a full cross section within only one exposure of a digital camera. The range of system sizes that RIMS can image is huge - this range is only limited from below by pixel resolution and laser sheet thickness. The imaging speed is mostly set by the amount of fluorescent light available. We have done successful imaging at an exposure time of $10 \mathrm{~ms}$ - this makes the imaging rate of RIMS on par with the fastest confocal scanners nowadays available. Another advantage of the RIMS technique is that it is cheap compared to the other techniques mentioned. The technology required is a standard workstation, a laser sheet on a translation stage, and a digital camera, so costs are about 
10-20 $\mathrm{k} \$$. In comparison, an MRI scanner costs on the order of $1 \mathrm{M} \$$, X-ray tomography scanners cost several $100 \mathrm{k} \$$, and a fast confocal 100-200 $\mathrm{k} \$$. These scanning techniques are also usually bought as stand-alone units and cannot easily be adapted to the requirements of the specific experiment; RIMS, with its simple components, can be almost freely adapted to any flow geometry.

Ordinary refractive index matched imaging has a long history: it has been applied to such diverse subjects as contrast variation scattering experiments with visible light, laser Doppler anemometry, blood flow anemometry, porous media flows, fluidized bed reactors, and ground water hydrology. ${ }^{19-33}$ In recent years, RIM has been combined with particle image velocimetry ${ }^{34}$ (PIV) and particle tracking velocimetry in two dimensions (2D PTV) for tracer particles ${ }^{28,35}$ and for the study of 3D flow fields. ${ }^{36,37}$ These techniques have been reviewed elsewhere. ${ }^{34,38-40}$

However, imaging and reconstructing the full threedimensional structure of granular packings and suspensions with an index matching technique has only recently become possible; see, for example, Slotterback ${ }^{29}$ and Huang. ${ }^{33}$ Also tracking particle motion with RIMS has become possible, e.g., in the case of three-dimensional continuous ${ }^{41-44}$ and cyclic shear. ${ }^{46-48}$ In this article, we will give a complete overview how to make a RIMS instrument that allows for three-dimensional particle tracking velocimetry (3D PTV).

Three-dimensional particle tracking places stringent demands on imaging methods: it is necessary to be able to uniquely identify particles and to distinguish them from close and often contacting neighbors. Additionally, it comes with limitations on size and shape of various components, and can require advanced imaging technology. Additionally, we discuss here the know-how on the technique and practical information typically not found in the literature. RIMS techniques overlap substantially with the regular RIM techniques. This review, therefore, serves to discuss both the particularities of RIMS, but is also useful for the general field of RIM imaging.

In Sec. II, we will give a brief description of the RIMS technique. In Sec. III, we will discuss the ingredients necessary for index matching, and we will discuss the techniques to achieve optimal index matching. In Sec. IV, we discuss the technical challenges in a typical RIMS setup, and present possible solutions. Finally, we give an outlook on the future of the RIMS technique.

\section{INDEX MATCHED SCANNING BASICS}

Refractive index matched scanning is a tomographic-like imaging technique and works as follows: one submerses particles in a fluorescent liquid with the same optical index. The dye, only present in the fluid, is excited by a laser sheet (see Fig. 1(a)). The fluorescent light from the fluid can be detected with a camera, and a typical camera image will show the particles as dark spots in a bright, well-defined cross section of the material (see Fig. 1(b)). The particles are visualized in three dimensions by moving the laser and simultaneously recording images to acquire image stacks, as shown in Fig. 1(c). These three-dimensional image stacks are obtained
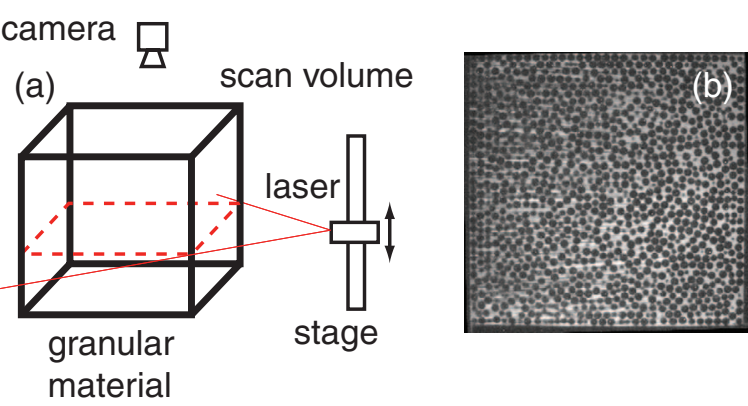

granular

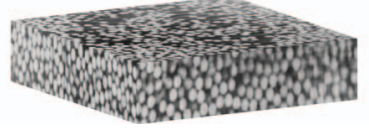

(c)

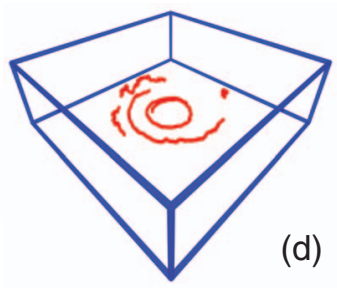

FIG. 1. (Color online) (a) A schematic overview of a RIMS setup, with all the essential components indicated. (b) A typical cross section of a suspension, obtained with RIMS. Particles (diameter $5 \mathrm{~mm}$ ) appear as dark spots in a bright background. (c) A stack of cross sections. Brightness is inverted for clarity. (d) From subsequent volume scans, one can obtain particle traces; a few examples are shown here as red/gray lines in the box. The flow is driven from the bottom by a rotating disk, hence the circular trajectories. The stochastic motion of the particles is clearly visible.

for subsequent time steps. Image processing techniques can then be used to extract particle positions and trajectories. Figure 1(d) shows typical examples of such trajectories. ${ }^{42}$ Note that suspensions of non-Brownian particles, when driven slowly enough, behave just like dry granular materials. ${ }^{52-54}$

A typical RIMS setup suitable for particle tracking uses particles with a diameter of typically $\sim$ millimeter. Typical scan volumes measure 10-30 particles across, so the typical scan volume $L^{3}$ is about 1 liter (see Fig. 2). The camera distance $r$ is typically about $50 \mathrm{~cm}$. The laser sheet is usually focussed on $f \sim 30 \mathrm{~cm}$. The laser sheet thickness $e$ should be less than a particle diameter $d$ and is typically a few hundred micrometers. Beam divergence in the laser sheet makes that

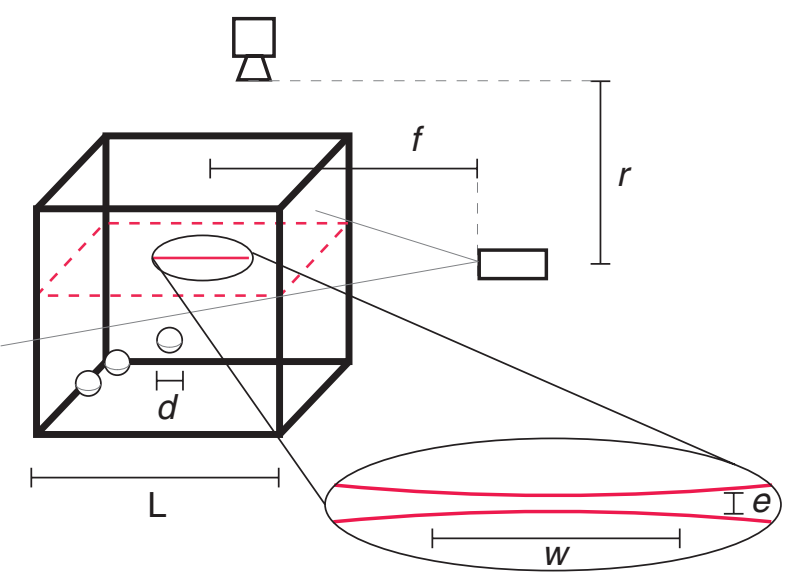

FIG. 2. (Color online) The lengthscales encountered in a RIMS setup. The scan volume size is $L$, the particle diameter is $d$, the laser sheet to camera distance is $r$. The focal point of the laser is at distance $f$, the sheet thickness is $e$, and the focusing width (see text) is $w$. 
the thickness of the sheet is not uniform; it is defined as the distance $w$ over which the thickness does not vary more than $\sqrt{2}$, also referred to as the Rayleigh range - this is usually about a few centimeters.

These lengthscales indicate that RIMS is useful for imaging centimeter-sized volumes, in which particles of millimeter size are being tracked. For particles smaller than $10 \mu \mathrm{m}$, confocal microscopy is more suitable ${ }^{18}$ (see the Introduction). As we will discuss below, scan speeds of up to a volume per second can be reached. This limits flow speeds that can be imaged with RIMS to about one particle diameter per second.

The design of a RIMS setup confronts one with two main challenges: $(i)$ how to achieve index matching? and (ii) how to capture stacks of images? In Secs. III and IV, we address both questions by reviewing the literature, and by describing our own methods.

\section{INDEX MATCHED SCANNING: MATERIALS AND METHODS}

To achieve index matching sufficient for RIMS, the difference in refractive indices of solid and liquid phase of the mixture needs to be less than $\pm 2 \times 10^{-3}$, as we will see below. Over the years, several recipes for combinations of index matching fluids and particles have been found. ${ }^{34,39,40,55-60}$ However, there is always some variation in the properties of commercially available products. Therefore, some degree of tuning the recipes to the individual experiment always remains necessary. We will give an overview of the most common solids and liquids available for index matching, the fluorescent dyes compatible with them, and will discuss their most relevant properties. We will also describe various techniques to fine tune index matching. A more detailed discussion of recipes can be found in Section 2 of the Appendix.

\section{A. Solids}

Solids tend to have a higher index of refraction than liquids. There are several transparent solids we know of that have a low enough index of refraction to use them for index matching, which are also available commercially as spheres. We list the properties of these materials in Table I. There are soda lime glass, crystal glass (also known as lead glass), fused silica glass, ${ }^{61}$ borosilicate glass, and the polymer poly(methyl metacrylate) (PMMA), also known as acrylic or plexiglas. The index of these materials ranges from 1.45 to 1.60 and is usually dependent on the manufacturer, ${ }^{62}$ except for the standard glass types such as BK7, which has well-defined properties. ${ }^{51}$ Hydrogel $^{4,63}$ is different in character. This polymer gel can come in the form of $\sim$ millimeter-sized spheres. It absorbs many times its initial volume in water, thereby becoming large, soft, and transparent, with an index of refraction comparable to that of water.

The refractive indices for most materials specified in this paper are for reference only; some authors claim they are well defined, ${ }^{64}$ but variations have also been reported. ${ }^{65-67}$ The index of refraction depends on temperature and wavelength (a phenomenon called dispersion) and is, therefore, customarily specified at the sodium D-lines at $589 \mathrm{~nm}$, at $20^{\circ} \mathrm{C}$; we will re-
TABLE I. Specifications of different kinds of transparent materials. The first five materials are all types of glass. BK7 glass is a borosilicate glass with well-defined properties. For more information on different glass types, see Refs. 49-51. Refractive indices as specified by manufacturers or commercial resellers. Price increase indicated is exponential, and given only for 3 $\mathrm{mm}$ spheres or closest available size. Besides size, the price also depends on supplier, sphericity, and optical quality (see Fig. 4).

\begin{tabular}{|c|c|c|c|c|}
\hline Material & $\begin{array}{c}\text { Index } \\
n_{D}\end{array}$ & $\begin{array}{c}\text { Diameter } \\
\text { range }[\mathrm{mm}]\end{array}$ & Price & Company \\
\hline Soda lime & $\sim 1.52$ & $0.1-10$ & ++ & Sigmund Lindner \\
\hline Crystal & $\sim 1.59$ & 3 & ++++ & Sandoz Fils SA \\
\hline Borosilicate & $\sim 1.5$ & $0.1-5$ & ++ & Sigmund Linder \\
\hline Fused silica & $1.45-1.46$ & $2-3$ & +++++ & Sandoz Fils SA \\
\hline $\mathrm{BK} 7$ & 1.5168 & $2-3$ & +++++ & $\begin{array}{l}\text { Worf Glaskugeln } \\
\text { GmbH }\end{array}$ \\
\hline PMMA & $1.47-1.50$ & $0.1-10$ & ++++ & $\begin{array}{l}\text { Engineering } \\
\text { Labs/Spherotech }\end{array}$ \\
\hline Hydrogel & $1.33-1.34$ & $10-100$ & + & $\begin{array}{l}\text { Educational } \\
\text { Innovations }\end{array}$ \\
\hline
\end{tabular}

fer to it with $\mathrm{n}_{D}$ from now on. Typically dispersion is stronger for fluids than for solids. The exact dispersion relation depends on the material; for a standard solid such as BK7, this is well specified. ${ }^{68}$ For an overview of dispersion relations for typical RIM materials, consider Refs. 58 and 69.

Optical homogeneity of glass beads varies by type and manufacturer. We have found that different batches of particles from the same source can have different refractive indices with variations up to 0.01 . Particles within one batch will have variations as well. Chemical compatibility is also a concern; PMMA is a polymeric solid known to absorb water. We have found that it also absorbs Triton X-100 and dimethyl sulfoxide (DMSO, $\left.\left(\mathrm{CH}_{3}\right)_{2} \mathrm{SO}\right)$. Absorption of liquids changes the index of refraction of PMMA particles by as much $^{70}$ as 2.5 $\times 10^{-3}$. Particle size is limited by commercial availability; very monodisperse sets of particles with $d<500 \mu \mathrm{m}$ are difficult to obtain, sieving a polydisperse set is then the only option. Monodisperse particles are easier to track, yet they have the tendency to crystallize ${ }^{71}$ which may impede some experiments.

\section{B. Liquids}

In Sec. III A, we have seen that for index matching of common solids a liquid with an index of at least 1.45 is required. Several candidates for liquids have been found ${ }^{39,40,55-60}$ we present a list of the main candidates in Table II. Besides the refractive index, there are other physical and chemical properties to consider. We list them in Section 1 of the Appendix.

\section{Fluorescent dyes}

When choosing a fluorescent dye, the peak in its absorption spectrum should be matched with the wavelength of the laser used. Besides this consideration and the price, there are other less obvious properties of laser dyes that are essential in RIM applications. See Table III for some examples of dyes. 
TABLE II. Table with common high $n_{D}$ fluids; (aq) means if dissolved in an aqueous solution. Refractive index data obtained from various commercial resellers and from Ref. 69.

\begin{tabular}{lc}
\hline \hline Solvent & $n_{D}$ (range) \\
\hline Triton X-100 & 1.49 \\
DMSO & 1.479 \\
1-Methylnaphthalene & 1.615 \\
Sodium poly tungstate (aq) & $1.33-1.55$ \\
Eugenol & 1.541 \\
NaI (aq) & $1.33-1.502$ \\
Methyl salicylate & 1.536 \\
CS & 1.627 \\
Cargille Labs' index matching liquids & $1.30-2.11$ \\
Cyclohexyl bromide & 1.495 \\
Glycerine & 1.474 \\
Sucrose (aq) & $1.33-1.49$ \\
para-Cymene & 1.49 \\
\hline
\end{tabular}

Stokes shift: The difference between the absorbed and the emitted wavelengths is called the Stokes shift. This shift is measured between the peaks of the absorption and emission spectra of the dye. On the one hand, the shift is large enough so that the two spectra do not overlap. This allows for filtering of scattered photons in the imaging with cheaper optical components as described in Sec. IV C. On the other hand, the Stokes shift should not be too large since index matching can only be tuned to one wavelength. To estimate the maximum tolerable Stokes shift, we assume that the deviation in the index between the solid and the liquid phase should be less than 0.002 (see Sec. III D). For example, BK7 glass has a dispersion of $\mathrm{d} n / \mathrm{d} \lambda=-0.4 \times 10^{-4} \mathrm{~nm}^{-1}$ and a typical liquid has a dispersion of $\mathrm{d} n / \mathrm{d} \lambda=-1 \times 10^{-4} \mathrm{~nm}^{-1}$ around $\lambda=600 \mathrm{~nm}$. Under these conditions, one calculates that a Stokes shift of more than $30 \mathrm{~nm}$ is not desirable.

The emission spectrum of the dye should be narrow for dispersion, and should cover the absorption spectrum of the light sensitive element used in the digital camera. Typically, digital cameras have their maximum sensitivity around 500$600 \mathrm{~nm}$. The quantum yield of the dye needs to be as high as possible, since absorption of photons increases the contrast gradient (see below). Photobleaching is the effect of the dye losing its capacity to fluoresce. This is a natural degradation process that is enhanced in the presence of (laser) light. For this reason, most laser dyes have to be stored in a dark environment. The sensitivity to photobleaching depends on the type of dye. The solvent can adversely affect the absorption

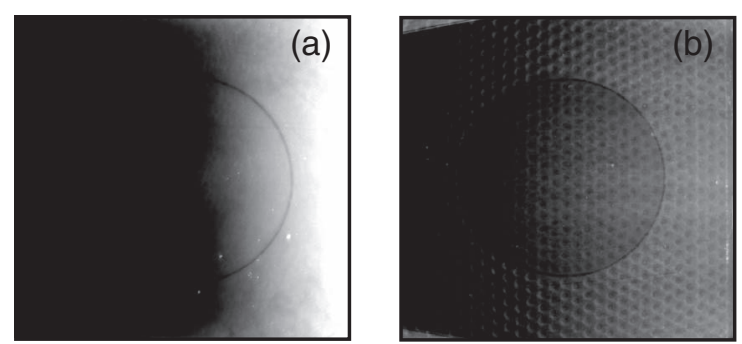

FIG. 3. Top views of a cross section of a RIMS volume. The laser sheet shines from the right and intersects a layer in which only the dyed fluid is present. (a) With a high dye concentration, the gradient in the fluorescence is clearly visible. (b) Using a lower dye concentration, the contrast is decreased, and even deep in the box, far to the left, fluorescence is still observed.

and emission spectra of laser dyes. Nile Blue 690 perchlorate is an example: ${ }^{72}$ it turns pink in pure Triton, but when water or $\mathrm{HCl}$ is added to the solvent, it becomes dark blue. Handling: Most fluorescent dyes come in the form of dry powder and are sold in milligram quantities. This makes it very hard to dose and mix them easily and safely - most fluorescent dyes have never been tested for their toxicity. Handling can be simplified, though: dyes can usually be dissolved in ethanol. In dissolved state, they still have a long shelf life (we have stored Nile Blue 690 perchlorate already for over two years under such conditions without visible deterioration of the dye). Dissolved in ethanol dyes can be safely handled and applied with a micropipette, and allow for easy mixing with the RIM liquid. The amount of ethanol added is usually so small that it has a negligible effect on the index of refraction; moreover, ethanol evaporates rapidly under most conditions.

The amount of laser dye in the RIM liquid balances two effects: with more dye present, the fluorescence will be brighter so the contrast between the dark particles and the bright fluid will be more pronounced. Additionally, more fluorescence allows for shorter exposure times. On the other hand, the presence of the dye will prevent the laser light from penetrating deep into the measurement volume; typically $I(x) \sim \exp (-\rho x)$, where $I(x)$ is the intensity of the laser light at distance $x$ from the source and $\rho$ is the dye concentration - this is usually referred to as Bouguer-Lambert-Beer's law. See Fig. 3 for an example of this effect. Note that the use of a laser line generator with a finite fan angle (see Sec. IV) will naturally produce an intensity gradient $I \sim A-B x$, with $A$ and $B$ constants, due to the broadening of the laser line; the exponential drop in intensity due to the presence of the dye comes in addition to this effect. Thus, a higher dye concentration leads to particle-fluid contrast inhomogeneities in the

TABLE III. Table with fluorescent dyes used. $\lambda_{\text {abs }}$ and $\lambda_{\text {emi }}$ are absorption peak and emission peak wavelengths; note that these wavelengths depend on the solvent the dyes are dissolved in. Only confirmed solvent compatibility is mentioned; compatibility with other solvents is not excluded. However, we found that Rhodamine $6 \mathrm{G}$ cannot be dissolved in NaI (aq) or in a mixture of Cyclohexyl bromide + Decalin. Unreferenced compatibilities we have tested ourselves. Dyes are available from, e.g., Exciton, American Dye Source, Radiant, Atto-tec.

\begin{tabular}{|c|c|c|c|}
\hline Dye & $\lambda_{\text {abs }}(\mathrm{nm})$ & $\lambda_{\text {emi }}(\mathrm{nm})$ & Compatibility \\
\hline Nile Blue 690 perchlorate & 633 & $650-690$ & Cargille type $\mathrm{DF}^{29} \mathrm{H}_{2} \mathrm{O}$, Triton ${ }^{54}$ \\
\hline Rhodamine $6 \mathrm{G}$ & 530 & $555-585$ & $\mathrm{DMSO}^{44}$ \\
\hline Atto 665 & 663 & 684 & $\mathrm{H}_{2} \mathrm{O}$, Cargille \# 19651 \\
\hline Pyrromethene 567A, 597-8C9 & 519,524 & 536,588 & 1-Methylnaphthalene \\
\hline
\end{tabular}



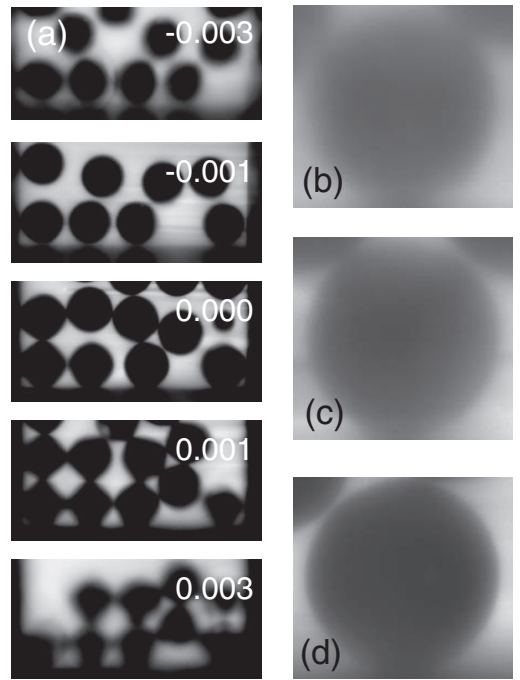

FIG. 4. (a) A cross section of BK7 glass spheres in a fluid with varying index of refraction (see text). The number indicates the index mismatch $\mathrm{n}_{f}-\mathrm{n}_{B}$ (see text) from the best matched sample (center). (b)-(d) Images of $3 \mathrm{~mm}$ glass beads at best index matching, $\sim 15$ layers deep, for (b) soda-lime glass, (c) crystal glass, (d) BK7 glass.

imaging volume, which can make imaging impossible, or may affect detection algorithms.

To optimize the dye contrast, the following rule of thumb seems reasonable to adhere to: Over the full width of the volume that is to be imaged the fluorescent intensity in the fluid phase should not drop more than a factor two. Note that the decay length of the fluorescence in the suspension is larger than that of the pure fluid (at the same dye concentration) by a factor $1 /(1-\phi)$ with $\phi$ the particle volume fraction, since transparent particles do not significantly absorb laser light. For different combinations of dye and liquid we found proper dye concentrations on the order of 0.1 to $10 \mathrm{mg} / \mathrm{l}$. Contrast gradients can also be reduced by introducing laser light in the same plane, but from the opposing side of the original laser. ${ }^{44}$ Note that sometimes light scattering effects can be used to visualize particles without the use of a dye. ${ }^{33}$

\section{Index matching: Quality and tuning}

The refractive index of liquids can be adapted to that of solids by using mixtures, but to what degree is index matching necessary? To illustrate how well particles and fluids must be index-matched, we look at a slice of a packing of $3 \mathrm{~mm} \mathrm{BK} 7$ glass spheres in a mixture of mineral oil and 1Methylnaphthalene in a $43 \mathrm{~mm}$ deep cuvette. Index tuning is achieved by adding droplets of mineral oil to the sample and subsequent stirring; the refractive index of the fluid was measured with an Abbe refractometer. We used a $532 \mathrm{~nm}$ laser to excite the Pyrromethene 567A dye dissolved in the fluid; the slice is imaged through about 15 particle layers (see Fig. 4(a)). The index mismatch is indicated by the mismatch $\mathrm{n}_{f}-\mathrm{n}_{B}$ between the fluid index $\mathrm{n}_{f}$ and the particle index $\mathrm{n}_{B}$. From this experiment, it is clear that to image through 15 particle layers, an index mismatch of 0.003 is about the largest tolerable: due to light scatter, the shape of the particles start to deviate from spherical, which makes detection increasingly difficult.

There is a limitation to how well index matching can be achieved: among particles of the same material and production batch, there are also small refractive index variations. We observe this by looking at a cross section at $\sim 15$ layers deep of a particle packing at best index matching, for three different types of glass (see Figs. 4(b)-4(d)). Clearly the BK7 glass gives the best contrast.

We can get further insight into index matching by using a ray-tracing algorithm (POV-Ray) to compute how the refraction of a non-index matched suspension of about 25 particle layers distorts the image of a red cone placed behind it (see Fig. 5). The tip of the cone sticks out above the suspension; the cone is observed through a sphere packing obtained from the experimental data ${ }^{42}$ (see Fig. 5(a)). In Fig. 5(b), the fluid has an index of $\mathrm{n}_{f}=1.500$. The figure suggests that a matching to at least $2 \times 10^{-3}$ is necessary to be able to image the 25 particle diameters. This is comparable to the index mismatch obtained in the experiments shown in Fig. 4, and similarly in Fig. 4(a) an asymmetry in index matching quality can be observed: for $\mathrm{n}_{f}-\mathrm{n}_{B}<0$, the effect of index mismatching seems to affect the cone (particle) shape less.

Ray tracing allows us to do tests not easily achieved in experiments: first of all, we can vary the spread in the index of refraction of the particle batch to mimic the effect observed in Figs. 4(b)-4(d): In Fig. 5(c), we show quantitatively how this spread affects the quality of the index matching. We give the particles an index of refraction from a normal distribution with mean particle index $\mathrm{n}_{B}=1.500=\mathrm{n}_{f}$ and standard deviation $\sigma=0.001$. Comparing the result to Fig. 5(b), we see that this spread gives a blur roughly equal to that of 0.002 index mismatch. We are not aware of any study that mentions how large the standard deviation in the index of refraction for typical glass particles is, although Ref. 49 mentions how accurate a refractive index can be defined for optical glass. Note that this type of image blur is inherent and can only be removed by changing particles. Second, we can see that the index tuning demands increase with the number of particle layers, ${ }^{74}$ as is shown in Fig. 5(d). Layer blur seems to increase roughly linearly with the number of layers, consistent with the observations of the fluorescent sheet index mismatch test shown in Fig. 4: that experiment gave an upper index mismatch limit of about 0.003 for 15 layers. In the ray-traced images, we see also considerable blurring of the cone imaged through a suspension of about 25 layers at an index mismatch of 0.002 .

The refractive index of most commercial products is not specified up to $0.2 \%$ as necessary for index matching. Overall variation in the mean index of refraction of bead batches makes tuning of liquid mixtures to the particles necessary. This is best done either in situ or cuvette, with the laser light that is also to be used in the actual experiment, to mitigate the effect of dispersion. In fact, the index of refraction of most materials also shows a temperature dependence. Generally speaking, the refractive index of liquids and solids goes down with increasing temperature. Typical temperature coefficients ${ }^{75,76}$ are in the range of $0.0005 \mathrm{~K}^{-1}$ for polymeric fluids, and $0.0001 \mathrm{~K}^{-1}$ for water. ${ }^{58} \mathrm{~A}$ temperature stability of at least $1^{\circ} \mathrm{C}$ is therefore essential. The temperature 
(a)
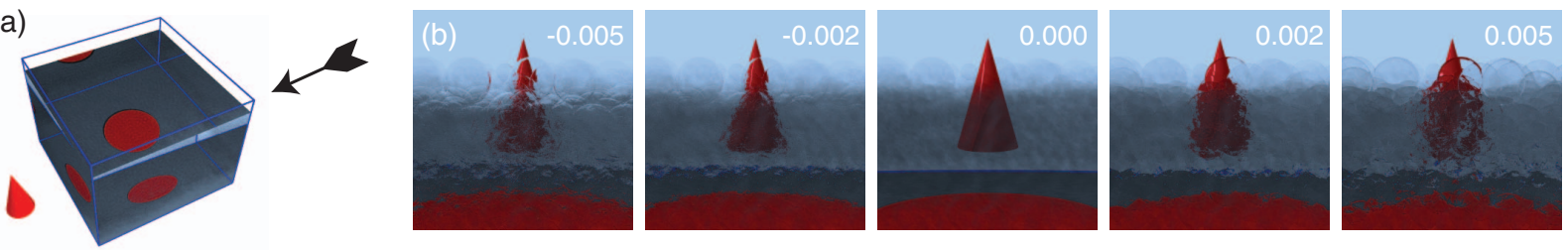

(c)
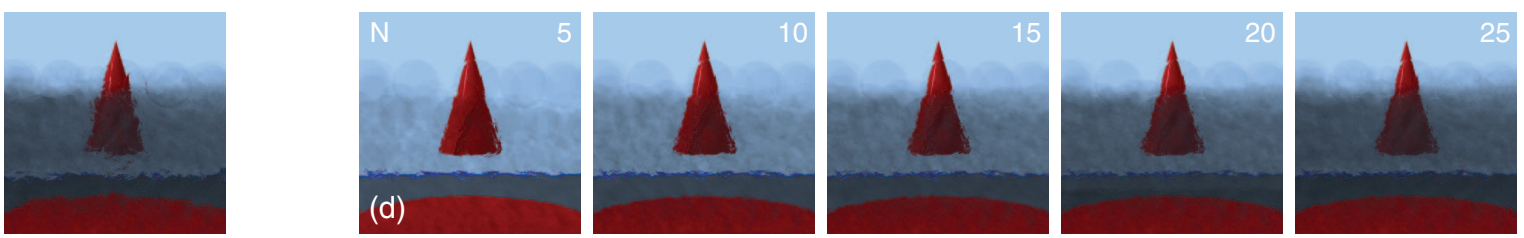

FIG. 5. (Color) Ray-traced images of the visibility of a red cone and a red disk buried under seven layers of particles, in a box with about 25 particle layers between cone and camera. (a) The perspective; the arrow indicates from which direction the cone is observed. (b) The effect of index mismatch $\mathrm{n}_{f}-\mathrm{n}_{B}$ by keeping the index of the fluid $\mathrm{n}_{f}=1.500$ constant. (c) The effect of a spread with standard deviation of 0.001 in the index of refraction of the beads; the mean $\mathrm{n}_{B}=\mathrm{n}_{f}=1.500$. (d) The effect of the number of layers $N$ imaged, with an index difference of 0.001 between the fluid and the particles and no index spread in the particles.

dependence of the index of refraction can also be used to fine tune the index of the liquid to the solid. ${ }^{33,34}$

There are several methods to fine-tune the index matching. Index mismatching can be observed and, thus, minimized via the backward reflection of the light of the laser sheet going through an immersed object, as discussed in Ref. 40. In Refs. 74 and 77, the transmittance per unit length of the particle suspension was shown to have a distinctive peak where index matching is best. Other methods are mentioned in Refs. 34 and 73.

We present here a different technique to measure the quality of index matching of the RIMS system. We shine a sheet of laser light through a cuvette filled with an RIM liquid and particles; the laser is pointing towards the camera. The light scatters off the suspensions, and this scattered light is captured on a milky semi-transparent plate. A camera then records the pattern on the milky plate while the RIM liquid is slowly diluted with a solvent. For example pictures, see Fig. 6 (I and II). From that image, we subtract a reference image, obtained by shining the laser line through a vial containing only the fluorescent fluid (Fig. 6(a)). The total differ-

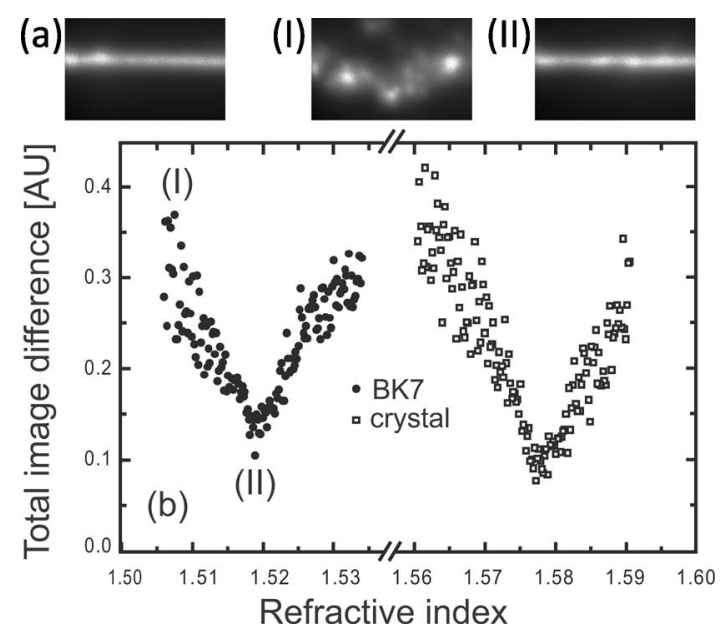

FIG. 6. (a) The reference image for index mismatch measurements (see text). (b) Measurements of the image distortion at different fluid indices. Image (I) refers to worse matching and (II) refers to best matching. ence between the reference image and the scattered image is then a measure for the quality of the index matching, and has a well-defined sharp minimum (see Fig. 6(b)).

Note that the ray-tracing studies above indicate that testing index matching in situ by means of testing the blurring of an object through the prepared suspension, for example, by estimating the readability of a text, should be a simple and reliable method as well.

\section{INDEX MATCHED SCANNING: INSTRUMENT DESIGN}

In this section, we discuss the various engineering details of RIMS imaging setups. We shall discuss sizing, imaging rates, video, illumination and optics, and mechanical components.

\section{A. Setup dimensions}

In Sec. II, we determined the typical size of a RIMS setup to be about 1 liter, with particles being about millimeter sized. These dimensions cannot be arbitrarily reduced or increased. What are the limiting factors?

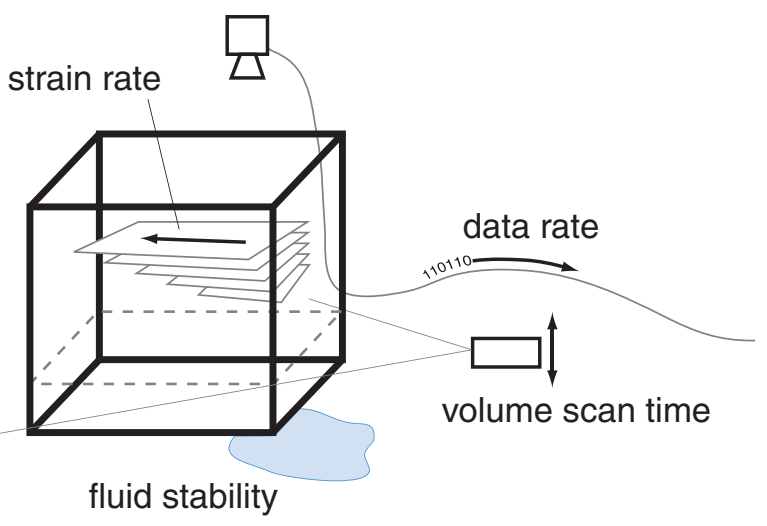

FIG. 7. (Color online) Various timescales encountered in RIMS setups. The strain rate is set by the type of experiment. Volume scan time should be small enough to image a whole volume before the strain becomes too large. The data rate is tied to the volume scan time and is limited by the camera system and other setup components, as discussed in the text. 
The wavelength of the laser light limits the thickness of the sheet and, therefore, the particle size. A pinhole technique such as used in confocal imaging (see below) can reduce this limitation, but the pinhole technique requires larger exposure times. Laser lines that create a sheet thickness $e$ of about $15 \lambda$ within a workable focussing width $w$ are commercially available. $^{78}$

The observable imaging volume is limited by the increasing scatter with increasing penetration depth. There is a maximum number of layers of particles $l_{s}$ the laser sheet can traverse without scattering too much. Additionally, there is a maximum number of layers of particles $l_{f}$ that the fluoresced photons can traverse to reach the camera. $l_{s}$ and $l_{f}$ cannot be both maximized simultaneously, since index matching must be done either at the wavelength of the laser or at the emission peak of the fluoresced photons. Typically, one may assume $l_{s}+l_{f}$ to be roughly constant. This total length can be $>30$ for good index matching. ${ }^{44,45} l_{s}+l_{f}$ itself can only be improved by better matching. However, this photon path length constraint can be geometrically relaxed by using two laser sources from opposing sides as discussed above, or by using two cameras imaging the volume from opposing sides.

\section{B. Imaging rate}

In designing a RIMS setup, the deformation- or displacement rate one would like to image is a crucial parameter, since it sets the imaging rate imaging rate (Fig. 7). The required imaging rate sets the maximum stage speed, video system requirement, laser power, lens properties, etc. If the displacement of a particle between two frames is larger than half the particle diameter $d / 2$, the identification of particles between subsequent frames becomes impossible. Therefore, after completion of two volume scans, the maximum displacements should be smaller than $d / 2$. Identifying particles in subsequent frames is necessary to trace the trajectory of a single particle from frame to frame and to measure trajectories as shown in Fig. 1(d). The amount of displacement per unit of time depends strongly on the type of experiment and can be less than $0.1 d$ per hour in a thermal cycling experiment ${ }^{29}$ or $10 d$ per second in fast flow. For slow deformation, a single scan may take up to tens of minutes; faster flows can require a scan time of less than $1 \mathrm{~s}$.

Some experiments involve cyclic shear ${ }^{28,46-48,79}$ in which the system is typically imaged only after (a number of) complete shear cycles. It is to be expected that after one shear cycle, particles return to a position very close to their original position. Then, it is not the deformation per unit of time that sets the scan time, but the time it takes to complete a shear cycle. Also, it is possible to correct for affine deformations in the displacement field by making clever use of the PIV techniques. ${ }^{80}$ That loosens the constraints on the imaging rate as well (see Sec. IV E).

Apart from the strain rate limitation, there are also other factors that favor faster scanning rates, such as evaporation of the liquid components, temperature drift, or dye bleaching.

\section{Scan rate limitations}

There are several experimental factors that limit the maximum scan rate. For a short volume scan time, the laser sheet has to be scanned faster through the material. For reasons discussed in Sec. IV C, this means that the camera has to be on a moving stage. Inertia and stage design then limit the maximum scan rate. The imaging system is another limiting factor on the imaging rate. The third important limiting factor on the imaging rate is that enough photons have to be available from the laser and fluorescence, since fast scanning requires short exposure times. The number of photons that reach the camera chip is controlled by several factors: laser power, fluid/particle absorption coefficients, dye concentration, quantum efficiency of the dye, camera distance, quantum efficiency of the chip, lens aperture, and camera exposure time. All these factors limit the speed of a RIMS system. The maximum imaging rate we have been able to achieve was with an exposure time of $10 \mathrm{~ms}$. We used a Basler A622f camera at ambient temperature and full gain with an F/1.4 $8 \mathrm{~mm}$ objective at roughly $30 \mathrm{~cm}$. We imaged a suspension of Triton X-100 and 5 mm PMMA spheres with Nile Blue 690 as a dye. Dye excitation came from a $25 \mathrm{~mW}$ laser sheet (Coherent $\mathrm{SNF}$ ) with a $30^{\circ}$ fan angle, positioned at a distance of about $30 \mathrm{~cm}$ from a $15 \times 15 \mathrm{~cm}$ container. The dye concentration was such that sufficient contrast between particles and liquid was achieved to image the whole container.

\section{Illumination, optics, and video}

In this section, we discuss the laser details, the optical components used, and the video system demands for RIMS systems.

\section{Lasers}

A typical RIMS setup uses a laser with about $50 \mathrm{~mW}$ of output power. More laser power increases the contrast, yet it enhances photobleaching of the fluorescent dye.

In choosing the laser wavelength, one has to consider the absorption spectrum of the transparent materials used in the setup: typically, PMMA absorbs strongly below $400 \mathrm{~nm}$. Also the availability of matching dyes, output power, and line generators are essential.

Ideally the thickness of a laser sheet is less than a tenth of a particle diameter. It should have a uniform thickness throughout the scan volume. However, most line generators do not generate such sheets. A typical line generator consists of a cylindrical lens, that deforms the circular Gaussian beam profile of a laser in an elliptic Gaussian beam profile, with strong intensity gradients at the edges. More sophisticated line generators ${ }^{78}$ can create more uniform laser sheets. However, beam divergence always limits the uniformity in flatness away from the focal point. Since the focal point is best placed in the center of the imaging volume (Fig. 2), it is helpful if the focus of the laser can be adjusted.

\section{Optics}

Since the imaging rate is typically a concern, large aperture lenses are favorable for a RIMS setup. They are 
easily available for the industrial C-mount camera typically used in RIMS setups; lenses with F/0.9 are neither expensive nor prohibitively heavy. Direct scatter of the photons from the laser beam creates intensity inhomogeneities in the captured images. Scattered light comes directly from the laser and can, therefore, be of much higher intensity than the fluorescence. To eliminate these scattered photons in the image, a filter can be used. Depending on the Stokes shift (see above), either a longpass filter ${ }^{81}$ or a bandpass or notch filter, tuned to the laser wavelength, has to be used.

\section{Video}

Image capturing with digital cameras is a turnkey nowadays. There is one crucial feature of video systems that has to be taken into account: the video data rate. If fast scanning is desired, both imaging rates of 10-1000 frames per second and long continuous recording capacity should be available. A minimal dataset consisting of $1003 \mathrm{D}$ volume scans of typical $256 \times 256 \times 256$ pixels resolution with 8 bit pixel depth yields about $1.7 \mathrm{~GB}$ of data. For a scan time of 1 volume per $10 \mathrm{~s}$, this yields a data rate of $1.7 \mathrm{MB} / \mathrm{s}$. Since the data rate grows with resolution cubed, the bit depth of most cameras is typically $>10$ bits, and the scan time could drop below $1 \mathrm{~s}$, the limit of the maximum write speed of a single hard drive (about $50 \mathrm{MB} / \mathrm{s}$ ) is soon reached. For such high speed, long recording applications, more specialized hardware than a simple workstation is required.

Another factor of relevance is the dark current of the photosensitive chip, which is independent of the framerate. The dark current is the anomalous "detection" of photons by the pixels on the CCD chip, even if no light reaches the photosensitive parts. The lower it is, the more the chip signal can be amplified without being hindered by noise. The dark current can be reduced by cooling the chip; cameras with this feature are commercially available.

\section{Mechanics}

To scan different cross sections of the suspension, it is necessary to move the laser sheet. The images have to stay in focus during the motion of the laser. There are two ways to achieve this: first, either the camera is placed at a large, fixed distance and optics is chosen such that the depth of field extends over the whole range of laser sheet position. Second, for faster scanning, another solution is more favorable. For higher frame rates, one needs as much light as possible and since the dye in the fluid fluoresces photons in random directions the camera should be close to the sheet. Moreover, a large aperture lens is typically used, since they capture more light. The large aperture lenses and small object distance, however, reduce the depth of field substantially, making it necessary that the camera moves with the laser sheet.

Note, however, that the total optical path length between the sheet and the camera sensor changes with the relative distances the light travels through the air and the optically denser liquid. The camera and the laser sheet, therefore, have to be moved by different amounts. As is shown in Section 4 of the Appendix, if the sheet moves by $\Delta s$, the camera motion has to be rescaled by the fluid index $n_{f}$; it has to be moved by $\Delta s / n_{f}$ to keep the optical path length constant. A proper choice of $f$ and aperture can keep the depth of field large enough to relax this constraint; the small distortion in calibration in the images that then remains can be corrected for in postprocessing.

\section{E. Routines for postprocessing RIMS images}

After obtaining an image as shown in Fig. 1(c), particle positions can be obtained by (a combination of) common image processing techniques, such as thresholding, convolution, pattern matching, and morphological operations. ${ }^{82,83}$ Subpixel approximation can improve most tracking techniques to \pm 0.1 pixel; algorithms are discussed in Refs. 80,84 , and 85. If applicable, well-developed routines such as the ones by Grier, Crocker, and Weeks ${ }^{85,86}$ can be used. The aforementioned routines also set some restraints on the pixel density: for a successful reconstruction of the particle positions, the images that build up the three-dimensional image stacks should be taken with a resolution of approximately 10 pixels per particle diameter (in all directions).

Other authors have expanded the array of tracking techniques for, e.g., fast flowing systems ${ }^{80}$ and polydisperse mixtures. ${ }^{87}$

\section{CONCLUSIONS AND OUTLOOK}

Index-matched flow imaging techniques are by now well established. We have shown how this technique can be developed for and applied to the study of dense granular materials. In this article, we combine our own knowledge obtained over many years of experience in building index-matched scanners. We hope this effort makes constructing RIM imaging setups easier for others, and stimulate further the development of the technique. This should, we hope, propel research in the exciting fields of granular materials, but also in, for example, suspension dynamics and porous medium flows.

The outlook for RIMS setups is excellent. Advances in computer and laser technology will make substantial development in this field possible. Where do we see the most likely developments? Bessel wave laser beams ${ }^{88}$ may be used to improve the number of layers imaged. Faster scanning is an obvious avenue. Using faster linear actuators or rotating mirror techniques to scan a three-dimensional volume will push the maximum scanning speed up to levels well beyond what any other scanning technique can accomplish. Using fluorocarbons ${ }^{34}$ to achieve index matching should be relatively simple due to their low index of refraction. We are not aware of any work that used fluorocarbon spheres for RIMS, but fibers have been used for RIM purposes. ${ }^{89}$ Another possible line of development is the combination of RIMS with, for example, diffusing-wave or other spectroscopic techniques. Spectroscopic techniques have had significant success in inferring motion inside a granular material, especially diffusive wave spectroscopy. ${ }^{90}$ These techniques complement RIMS: They do not provide trajectories for all the particles, but can provide information with much higher time resolution.

Making RIMS setups fit for smaller particle size is another significant challenge. Especially the regime of 
$10-100 \mu \mathrm{m}$ particles is difficult to visualize: currently it is too big for confocal, too small for laser scanning.

\section{ACKNOWLEDGMENTS}

We would like to thank several people who over the years have stimulated us with their invaluable comments, help, ideas, and tips. J. C. Tsai and Herve Capart kindly shared their knowledge of RIM setups with us, and Matthias Schröter has been supportive in sharing his knowledge of index-matched scanning, and in being a critical reader of this manuscript. Peter Schall is acknowledged for support and sharing ideas. Elie Wandersman has found the best recipe to match PMMA particles to Triton mixtures, has been a critical user of the technique, and provided the data for Fig. 1. Masahiro Toiya has developed the first RIMS setup at the University of Maryland, where Steven Slotterback, who kindly shared his knowledge with us, continued working on it. We thank Markus Benderoth for pointing out the hazardousness of 1-Methylnaphthalene. Liane Hilfert and Sabine Busse carried out the NMR and mass spectroscopy experiments, respectively, on the Cargille oil.

This work was supported by NSF-DMR0907146, NSFDMR0906908, ARO-W911NF-11-1-0110, German research grant DFG STA 425/24, and the Dutch physics foundation FOM.

\section{APPENDIX: RIM LIQUID SPECIFICS AND OPTICAL PATH LENGTH CORRECTION}

In this Appendix, we discuss in more detail several indexmatching liquids and their properties and recipes, to provide the reader with a better understanding of the advantages and disadvantages of the various RIM recipes available. We also detail how the motion of the camera and the laser sheet should be corrected for the optical path between them to remain constant.

\section{Index matching liquids properties}

Here, we list a number of physical and chemical properties of RIM liquids that are of concern.

Safety: As an extreme example, the fluid $\mathrm{CS}_{2}$ can be mentioned: its index of refraction is 1.62 , allowing for index matching of a wide range of transparent solids. It is, however, extremely flammable and toxic, and for those reasons not to be used as a RIM component. Methyl salicylate is toxic if ingested, but otherwise relatively safe. Eugenol, DMSO, and various petrochemicals can give off irritant, nauseating, or toxic fumes, and so use of ventilation is recommended or required. Triton X-100 and sodium poly tungstate (SPT) are relatively safe when adhering to standard laboratory practice.

Hygroscopicity: DMSO is hygroscopic and in an open environment will take up water from the air, which will affect its refractive index.

Vapor pressure: A low vapor pressure liquid or liquid component requires sealed containers since different components can have different evaporation rates, which causes a drift in the composition of the liquid.
Stability: Chemicals may degrade by exposure to air or setup components; Eugenol slowly oxidizes and should be used in a closed container.

Corrosiveness: The chemical compatibility of RIM liquids with the various metals, plastics, rubbers, and epoxies present in a RIMS setup should be verified. For example, Eugenol reacts with plain steel and brass ${ }^{91}$ but not with aluminum, yet SPT slowly reacts with aluminum. Some polymeric fluids dissolve PMMA or other plastics. PMMA tends to absorb water and other liquids (see also Section 2 of the Appendix).

Dye compatibility: Miscibility is an obvious sine qua non, but also the fluorescence efficiency and spectrum of fluorescent dyes can be affected by the RIM liquid they are dissolved in (see, e.g., Ref. 72).

Viscosity can be too high to achieve index matching because it inhibits mixing and, therefore, tuning of the RIM liquid. For example, sucrose solutions can reach a very high index of refraction; Cargille offers standard sucrose solutions (product number 19259-BXS) with $n_{D}=1.49$. However, these solutions contain $80 \%$ sucrose, and have a viscosity ${ }^{92}$ of 20 Pa s.

The rheology of RIM liquids can be non-Newtonian. The above-mentioned sucrose solutions are Newtonian up to high concentrations and standard temperature, ${ }^{92}$ but, for example, Triton X-100 becomes non-Newtonian at temperatures below about ${ }^{93} 20^{\circ} \mathrm{C}$ and becomes a gel when mixed with sufficient amounts of water. ${ }^{94}$ See the following section for a more detailed overview of concerns regarding some common RIM liquids and recipes.

\section{Index matching recipes}

Over the years, many recipes to index match solids with fluids have emerged. Here, we will list a few index matching recipes that we have found to work or know from the literature. More recipes can be found in Refs. 39, 40, 55-60, 95, and 96; the most complete overview is in Ref. 34. For a large database of refractive indices and dispersion relations of solids and liquids, see Refs. 49 and 69. A number of patents also discuss safe high refractive index oils (see, for example, Ref. 97).

As mentioned before, recipes cannot be simply copied and expected to work without further tuning. For example, even for Triton X-100 based RIM mixtures, several different recipes exist in the literature, as we will discuss below.

Triton $X-100$ is a polymeric fluid, with a viscosity of $0.22 \mathrm{~Pa}$ s. It mixes slowly with water; mixing is enhanced by adding $\mathrm{ZnCl}_{2}$. Drawbacks are its complex rheological behavior: it has a glass temperature around $5^{\circ} \mathrm{C}$ and becomes strongly non-Newtonian at temperature below ${ }^{93} 20^{\circ} \mathrm{C}$. Mixing it with $\mathrm{H}_{2} \mathrm{O}$ increases its viscosity and induces gelation at $50 \% \mathrm{H}_{2} \mathrm{O}$, an effect that is inhibited by adding salt. ${ }^{94}$ Its cloud point is $65^{\circ} \mathrm{C}$, and it is relatively difficult to clean. Reference 57 states the following relative quantities that can be used for index matching with Triton X-100: 77.9\% Triton, $13 \% \mathrm{H}_{2} \mathrm{O}, 9 \% \mathrm{ZnCl}_{2}$, all fraction by weight. This mixture was mentioned also to be density matched with the $0.1 \mathrm{~mm}$ PMMA particles; note that for $3 \mathrm{~mm}$ PMMA 
particles, this was not the case. ${ }^{93}$ The particles used in Ref. 57 were substantially smaller than $3 \mathrm{~mm}$, so most likely made with a different process. Also, that mixture was cloudy. ${ }^{93}$ In Ref. 95, we find a different composition of Triton, water, and zinc chloride used to index match PMMA: 92\% Triton, $4.67 \% \mathrm{H}_{2} \mathrm{O}, 3.22 \% \mathrm{ZnCl}_{2}$, and $0.1 \% 12 \mathrm{M} \mathrm{HCl}$. Breedveld ${ }^{96}$ mentions yet another fluid composition. The index matching in Ref. 42, on which also Figs. 1(b) and 1(c) are based, was made only with Triton X-100 and $2 \mathrm{ml}$ of a $37 \% \mathrm{HCl}$ solution added on every liter of Triton. Adding $\mathrm{HCl}$ to a Triton mixture is advantageous for two reasons: in case $\mathrm{ZnCl}_{2}$ and water are in the mixture as well, it helps to prevent the formation of white $\mathrm{Zn}(\mathrm{OH})_{2}$ crystals, which after a few days begin to precipitate out of the solution. $\mathrm{HCl}$ can also be used to tune the absorption and emission spectrum of a widely used fluorescent dye, Nile Blue 690 perchlorate (see Sec. III C). In Ref. 98, UCON oils and Triton X-100 were mixed with 1,6-dibromohexane to obtain index matching with PMMA. We have also observed that washing and drying PMMA particles after having submersed them in Triton X-100 often makes them develop cracks. Therefore, once submersed in Triton, PMMA particles are best stored submersed.

$D M S O$ is a low viscosity fluid with presumably Newtonian rheological behavior in any realistic RIM setting. However, PMMA particles are only stable in DMSO for a couple of days; after that, the fact that they absorb DMSO becomes noticeable in cracking and swelling of the particles. Furthermore, it is hygroscopic, and since dissolved water will change the refractive index of the mixture, it is best used in closed setups. The refractive index of PMMA can be matched using DMSO with a concentration of $0.1 \mathrm{~g} / \mathrm{ml} \mathrm{NaI}$.

$\mathrm{NaI}$ dissolved in water, without using DMSO as mentioned directly above, can reach an index of refraction ${ }^{56}$ up to 1.50. The solution has a yellow color, but this can be suppressed by adding $\mathrm{Na}_{2} \mathrm{SO}_{3}$. However, a high concentration of salt is needed to match the index of refraction of PMMA, which makes the density of the liquid much larger than that of the particles. In addition, the salt strongly quenches the fluorescence of the popular dye Rhodamine. Note that the silicate glasses used in RIM are known to leak alkali ions when submersed in aqueous solution, ${ }^{99}$ which can change their optical properties over time.

Ammonium thiocyanate can be mixed with glycerin to index match PMMA particles. This is a relatively safe and low viscosity recipe, and has been tested in RIM applications. ${ }^{74}$

1-Methylnaphthalene is a transparent and presumably toxic ${ }^{100}$ liquid with an index of 1.615 and, therefore, suitable for use with many different types of glass. To adjust a lower index, it can be mixed with turpentine ${ }^{28}$ or light mineral oil. ${ }^{48}$ The components evaporate very slowly and the index is sufficiently constant for at least two weeks. Disadvantages are its strong aromatic odor, necessitating air ventilation, and its limited solubility, making cleaning processes difficult.

Cyclohexyl bromide can be mixed with cis-decalin to index and density match PMMA. ${ }^{35}$ It is relatively safe and cheap, but has never been tested within a RIMS setting; it is more common for confocal imaging applications. ${ }^{101}$ Also, an aqueous solution of ammonium thiocyanate is mentioned ${ }^{74}$ as a relatively safe index matching component.
Note that several authors report on index matching borosilicate glass with glycerol $\left(n_{D}=1.474\right)$, although this was only done for suspensions with a low volume fraction of solid particles. See Ref. 34 for references. We have not tested whether this index matching is sufficient for dense suspensions, but if so, the availability and safety of glycerol would make it an ideal candidate.

Cargille oils can be bought with any specified index of refraction between 1.300 and 2.110 , and have been used successfully in RIMS applications..$^{29,47}$ However, the volumes typically necessary in RIMS setups make them quite expensive for RIM purposes. Moreover, they can be toxic. The exact composition of these liquids is not publicly known, so we performed nuclear magnetic resonance (NMR) spectroscopy and mass spectroscopy analysis on an immersion liquid from Cargille $\left(\mathrm{n}_{D}=1.52\right.$, code 1160 , catalog number 19561). This revealed that they are a mixture of benzyl-butyl-phtalate and di-octyl-phtalate, and possibly decanol.

Para-cymene has been used in combination with PMMA; ${ }^{33}$ index matching was achieved by tuning the temperature of the fluid. Para-Cymene slowly dissolves PMMA, so particles had to be washed after each experiment. Unlike with Triton, repeated washing and drying does not lead to disintegration of the particles. ${ }^{102}$

Eugenol or clove oil is a natural product with a high index of refraction and low viscosity. It is light brown. Its drawbacks are that it is incompatible with steel and brass ${ }^{91}$ and some plastics, and oxidizes very quickly, which changes its color and index of refraction.

There are also certain high refractive index liquids that have, to our knowledge, not yet been tested in a RIM settings, but which have properties that makes them attractive candidates for this purpose: Methyl salicylate, also known as oil of wintergreen, has a high index of refraction and mixes well with water and ethanol. However, it is known to be toxic if ingested in small quantities. The chemically related material ethyl salicylate may be less harmful. SPT solutions can achieve moderately high indices, making index matching with materials like polystyrene $\left(n_{D}=1.55\right)$ and other more common types of glass possible. This salt is compatible with most metals (except aluminum), glass, and PMMA. However, very high concentrations of the salt are necessary to achieve this high index, and high concentration SPT solutions have a density higher than that of PMMA and most glasses. Moreover, its viscosity increases dramatically with concentration and its rheological behavior at such high salt concentrations is not known.

\section{Density matching}

Density matching can be combined with index matching. ${ }^{34}$ In general, a three component fluid is necessary to tune both index of refraction and density unless one finds a two-component fluid that, for some ratio of their ingredients, has the same index and density as that of the particles used. The references we are aware of that give working recipes are Krishnan, ${ }^{57}$ Zarraga, ${ }^{95}$ and Breedveld, ${ }^{96}$ all for index matching with $\sim 100 \mu \mathrm{m}$ particles. These authors use PMMA particles, the first three use a mix of Triton X-100, 
$\mathrm{ZnCl}_{2}, \mathrm{HCl}$, and water. Curiously all papers report different relative quantities of the constituents. Reference 98 mixes UCON oils with Triton X-100 and 1,6-dibromohexane to obtain index and density matching, although this was used for laser Doppler velocimetry, and not for RIMS.

DMSO, NaI, and water can achieve density and index matching with $\mathrm{PMMA}^{44}$ by adding a small amount of $\mathrm{NaI}$ (aq) with a concentration of $1.6 \mathrm{~g} / \mathrm{ml}$ to the solvent. As mentioned before, this recipe is not stable for more than a few days due to the adverse effects of DMSO on PMMA. This is partly problematic since fluorescence of standard dyes is greatly reduced in this mixture, necessitating long exposure times and, therefore, long experimental timescales. The same question arises with the recipe from Ref. 74 - it has yet to be tested how well this recipe works for RIMS settings. Wang ${ }^{35}$ used cyclohexyl bromide mixed with cis-decalin to index and density match PMMA sufficiently to do 2D PTV with tracer particles. This mixture is commonly used for confocal microscopy. ${ }^{101}$

Density and index matching is more difficult with glass due to the higher density of most glasses. The only candidate for this, so far untested, is SPT, which can reach specific gravities of $>3$ and refractive indices of up to 1.55 .

\section{Optical path length correction}

The optical path length between the laser sheet and the camera varies with the laser sheet moving through the fluid, since the geometric path consists of changing fractions of media with different refractive indices. Along the optical axis, the trivial way to correct for this is to reduce the motion of the camera with respect to the laser sheet by a factor $n_{1}$, the refractive index of the liquid. In general, however a RIM setup is a complex optical system as there are different refractive indices and optical devices, and not all rays pass through the optical axis. Therefore, away from the optical axis the correction may be more complicated. To see if the off-axis path changes by a different amount, we calculate the optical path length between sensor and laser sheet by ray transfer matrices. ${ }^{103}$ At any point, a beam can be described by the distance $y$ and angle $\alpha$ relative to the optical axis (see Fig. 8(a)). The fluorescent light emitted from the illuminated plane propagates a distance $s_{1}$ until it is refracted by the transparent flat side wall of the container (see Fig. 8(b)). Then, the beam passes through the wall medium with thickness $s_{2}$ and is again refracted at the other side of the container wall. After a distance of $s_{3}$, the light is focused by the camera objective, here assumed to be a thin lens with focal length $f$. After translating $s_{4}$, the ray hits the camera sensor.

The ray path in this optical system can be described by multiplication of transfer matrices. The $2 \times 2$ matrices for the modification by translation $T_{i}$, refraction $R$, and a lens $O$ respectively, are ${ }^{103}$

$$
T_{i}=\left[\begin{array}{cc}
1 & \frac{s_{i}}{n_{i}} \\
0 & 1
\end{array}\right] ; \quad R=\left[\begin{array}{cc}
1 & 0 \\
0 & 1
\end{array}\right] ; \quad O=\left[\begin{array}{cc}
1 & 0 \\
-\frac{1}{f} & 1
\end{array}\right] .
$$

Only the translation matrix depends on the refractive index. In the following, we use the slope of the beam $v_{i}$ (a)

(b)
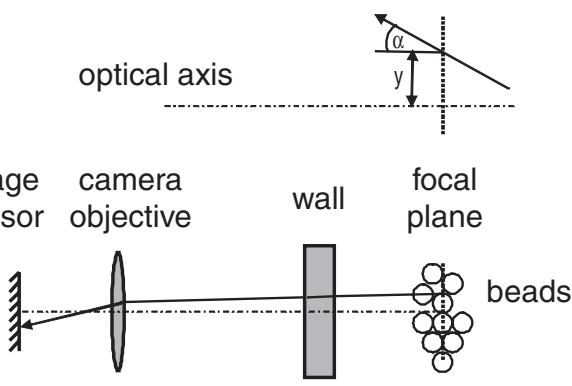

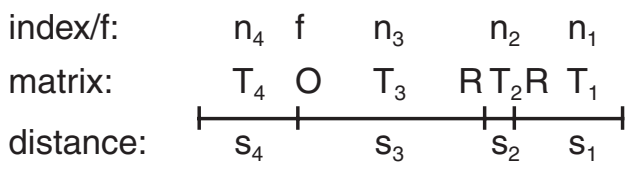

FIG. 8. (a) Any ray in an optical system is described by its distance to the optical axis and angle. (b) A schematic diagram of the optical elements of a general RIMS.

$=n_{i} \tan \left(\alpha_{i}\right)$ instead of its angle $\alpha_{i}$. We assume throughout that $n_{3}, n_{4}=1$. A ray that is emitted at distance $y_{1}$ and slope $v_{1}$ from the illuminated sheet hits the camera sensor at distance $y_{4}$ and slope $v_{4}$ by the relation

$$
\left[\begin{array}{l}
y_{4} \\
v_{4}
\end{array}\right]=T_{4} \cdot O \cdot T_{3} \cdot R \cdot T_{2} \cdot R \cdot T_{1}\left[\begin{array}{l}
y_{1} \\
v_{1}
\end{array}\right] .
$$

The matrices must then be multiplied in their physical order, here from right to left (see also Fig. 8(b)). This yields

$$
\left[\begin{array}{l}
y_{4} \\
v_{4}
\end{array}\right]=\left[\begin{array}{cc}
1-\frac{s_{4}}{f} & \left(1-\frac{s_{4}}{f}\right) \cdot c+s_{4} \\
-\frac{1}{f} & 1-\frac{c}{f}
\end{array}\right]\left[\begin{array}{l}
y_{1} \\
v_{1}
\end{array}\right],
$$

with $c=\frac{s_{1}}{n_{1}}+\frac{s_{2}}{n_{2}}+s_{3}$. The distance $s_{1}$ changes during the experiment to enable scanning. Only $s_{3}$ can be adjusted in order to compensate the optical path. We want to map the sheet plane one-to-one to the image sensor plane independent of $v_{1}$, so the top-right matrix element in the last equation must be zero. ${ }^{104}$ This must be so before and after motion of sheet and camera. Under this condition, we find that for any laser displacement $\Delta s_{1}$, the camera must be shifted by the factor $\Delta s_{3}$ $=-\Delta s_{1} / n_{1}$ decreasing (increasing) the geometric path length of $s_{1}$ if $s_{3}$ increases (decreases) to stay in the focal plane.

${ }^{1}$ Y. Forterre and O. Pouliquen, Annu. Rev. Fluid Mech. 40, 1 (2008).

${ }^{2}$ I. Goldhirsch, Annu. Rev. Fluid Mech. 35, 267 (2003).

${ }^{3}$ H. M. Jaeger, S. R. Nagel, and R. P. Behringer, Rev. Mod. Phys. 68, 1259 (1996).

${ }^{4}$ M. Iskander, Modelling with Transparent Solids (Springer, Berlin, 2010).

${ }^{5}$ J. Duran, Sands, Powders and Grains: An Introduction to the Physics of Granular Materials (Springer, New York, 2000).

${ }^{6}$ G. T. Seidler, G. Martinez, L. H. Seeley, K. H. Kim, E. A. Behne, S. Zaranek, B. D. Chapman, S. M. Heald, and D. L. Brewe, Phys. Rev. E 62, 8175 (2000).

${ }^{7}$ T. Aste, M. Saadatfar, and T. J. Senden, Phys. Rev. E 71, 061302 (2005).

${ }^{8}$ P. Richard, P. Philippe, F. Barbe, S. Bourlès, X. Thibault, and D. Bideau, Phys. Rev. E 68, 020301 (2003).

${ }^{9}$ M. Saadatfar, A. P. Sheppard, T. J. Senden, and A. J. Kabla, J. Mech. Phys. Solids. 60, 55 (2012).

${ }^{10}$ P. Philippe and D. Bideau, EPL 60, 677 (2002).

${ }^{11}$ M. Scheel, R. Seemann, M. Brinkmann, M. D. Michiel, A. Sheppard, B. Breidenbach, and S. Herminghaus, Nature Mater. 7, 189 (2008). 
${ }^{12}$ X. Cheng, J. B. Lechman, A. Fernandez-Barbero, G. S. Grest, H. M. Jaeger, G. S. Karczmar, M. E. Möbius, and S. R. Nagel, Phys. Rev. Lett. 96, 038001 (2006).

${ }^{13}$ K. Sakaie, D. Fenistein, T. J. Carroll, M. van Hecke, and P. Umbanhowar, EPL 84, 49902 (2008).

${ }^{14}$ N. Huang, G. Ovarlez, F. Bertrand, S. Rodts, P. Coussot, and D. Bonn, Phys. Rev. Lett. 94, 028301 (2005).

${ }^{15}$ Strictly speaking any non-magnetic material with a non-zero nuclear spin will suffice.

${ }^{16}$ Though the index matching does not have to be very well tuned as scatter of visible light with $\lesssim$ micrometer-sized particles is not very much affected by interfaces with slightly different indices of refraction.

${ }^{17}$ J. Zhou, S. Long, Q. Wang, and A. D. Dinsmore, Science 312, 1631 (2006).

${ }^{18}$ M. Toiya, J. Hettinga, and W. Losert, Granular Matter 9, 323 (2007).

${ }^{19}$ W. Johnston, A. Dybbs, and R. Edwards, Phys. Fluids 18, 913 (1975).

${ }^{20}$ A. K. V. Helden and A. Vrij, J. Colloid Interface Sci. 78, 312 (1980).

${ }^{21}$ A. K. V. Helden and A. Vrij, J. Colloid Interface Sci. 76, 418 (1980).

${ }^{22}$ B. J. Ackerson and P. N. Pusey, Phys. Rev. Lett. 61, 1033 (1988).

${ }^{23}$ S. E. Paulin and B. J. Ackerson, Phys. Rev. Lett. 64, 2663 (1990).

${ }^{24}$ C. Montemagno and W. Gray, Geophys. Res. Lett. 22, 425, doi:10.1029/94GL02697 (1995).

${ }^{25}$ M. Northrup, T. Kulp, S. Angel, and G. Pinder, Chem. Eng. Sci. 48, 13 (1993).

${ }^{26}$ A. Zachos, M. Kaiser, and W. Merzkirch, Exp. Fluids 20, 229 (1996).

${ }^{27}$ C. Egelhoff, R. Budwig, D. Elger, T. Khraishi, and K. Johansen, J. Biomech. 32, 1319 (1999).

${ }^{28}$ O. Pouliquen, M. Belzons, and M. Nicolas, Phys. Rev. Lett. 91, 014301 (2003).

${ }^{29}$ S. Slotterback, M. Toiya, L. Goff, J. F. Douglas, and W. Losert, Phys. Rev. Lett. 101, 258001 (2008).

${ }^{30}$ J. K. Arthur, D. W. Ruth, and M. F. Tachie, J. Fluid Mech. 629, 343 (2009).

${ }^{31}$ C. Stoots, S. Becker, K. Condie, F. Durst, and D. McEligot, Exp. Fluids 30, 391 (2001).

${ }^{32}$ M. M. Fontenot and R. D. Vigil, J. Colloid Interface Sci. 247, 481 (2002).

${ }^{33}$ A. Huang, M. Huang, H. Capart, and R.-H. Chen, Exp. Fluids 45, 309 (2008).

${ }^{34}$ S. Wiederseiner, N. Andreini, G. Epely-Chauvin, and C. Ancey, Exp. Fluids 50, 1183 (2011).

${ }^{35}$ P. Wang, C. Song, C. Briscoe, and H. A. Makse, Phys. Rev. E 77, 061309 (2008).

${ }^{36}$ J.-C. Tsai, G. A. Voth, and J. P. Gollub, Phys. Rev. Lett. 91, 064301 (2003).

${ }^{37}$ A. V. Orpe and A. Kudrolli, Phys. Rev. Lett. 98, 238001 (2007).

${ }^{38}$ F. Hendriks and A. Aviram, Rev. Sci. Instrum. 53, 75 (1982).

${ }^{39}$ B. Yip, M. Miller, A. Lozano, and R. Hanson, Exp. Fluids 17, 330 (1994).

${ }^{40}$ R. Budwig, Exp. Fluids 17, 350 (1994).

${ }^{41}$ K. B. Zitoun, S. K. Sastry, and Y. Guezennec, Int. J. Multiphase Flow 27, 1397 (2001)

${ }^{42}$ E. Wandersman, J. A. Dijksman, and M. van Hecke, (unpublished).

${ }^{43}$ J. A. Dijksman, E. Wandersman and M. van Hecke, Chaos 20, 041105 (2010).

${ }^{44}$ K. Lőrincz and P. Schall, Soft Matter 6, 3044 (2010).

${ }^{45}$ Wiederseiner (Ref. 34) can see through up to 175 particle diameters with sufficient matching for 2D PIV.

${ }^{46}$ A. Panaitescu and A. Kudrolli, Prog. Theor. Phys. Suppl. 184, 100 (2010).

${ }^{47}$ A. Panaitescu and A. Kudrolli, Phys. Rev. E 81, 060301 (2010).

${ }^{48}$ F. Rietz and M. Schröter (unpublished).

${ }^{49} \mathrm{H}$. Bach and N. Neuroth, Properties of Optical Glass (Springer, Berlin, 1998).

${ }^{50}$ M. Wakaki, K. Kudo, and T. Shibuya, Physical Properties and Data of Optical Materials (CRC Press, Boca Raton, 2007).

${ }^{51}$ M. J. Weber, Handbook of Optical Materials (CRC Press, Boca Raton, 2002).

${ }^{52}$ T. Divoux and J.-C. Géminard, Phys. Rev. Lett. 99, 258301 (2007).

${ }^{53}$ M. Pailha and O. Pouliquen, J. Fluid Mech. 633, 115 (2009).

${ }^{54}$ J. A. Dijksman, E. Wandersman, S. Slotterback, C. R. Berardi, W. D. Updegraff, M. van Hecke, and W. Losert, Phys. Rev. E 82, 060301 (2010).

${ }^{55}$ M. Cui and R. Adrian, Exp. Fluids 22, 261 (1997).

${ }^{56}$ T. Narrow, M. Yoda, and S. Abdel-Khalik, Exp. Fluids 28, 282 (2000).

${ }^{57}$ G. P. Krishnan, S. Beimfohr, and D. T. Leighton, J. Fluid Mech. 321, 371 (1996).

${ }^{58}$ M. Stöhr, K. Roth, and B. Jähne, Exp. Fluids 35, 159 (2003).

${ }^{59}$ P. Miller, K. Danielson, G. Moody, A. Slifka, E. Drexler, and J. Hertzberg, Exp. Fluids 41, 375 (2006).
${ }^{60}$ Y. A. Hassan and E. E. Dominguez-Ontiveros, Nucl. Eng. Des. 238, 3080 (2008).

${ }^{61}$ Fused silica is expensive to obtain as spherical particles, but if the shape of individual granules need not be spherical, then it is much cheaper, and an attractive option with $\mathrm{n}_{D}$ of $\sim 1.46$.

${ }^{62}$ Glass sphere manufacturer Sigmund Lindner calculates the index of the glass from the chemical composition, and does not measure it in the final product. Therefore, tolerances can arise. Private communication, 2011.

${ }^{63}$ S. Mukhopadhyay and J. Peixinho, Phys. Rev. E 84, 011302 (2011).

${ }^{64} \mathrm{~F}$. Träger, (Ed.), Springer Handbook of Lasers and Optics (Springer, New York, 2007).

${ }^{65}$ R. M. Waxler, D. Horowitz, and A. Feldman, Appl. Opt. 18, 101 (1979).

${ }^{66}$ S. N. Kasarova, N. G. Sultanova, C. D. Ivanov, and I. D. Nikolov, Opt. Mater. 29, 1481 (2007).

${ }^{67}$ M. Agelinchaab, M. F. Tachie, and D. W. Ruth, Phys. Fluids 18, 017105 (2006).

${ }^{68}$ See Schott, http://www.us.schott.com for properties of (optical) glasses.

${ }^{69} \mathrm{See}$ http://refractiveindex.info for dispersion relations of typical fluid and solid optical materials.

${ }^{70}$ Y. Ren, Opt. Mater. 19, 443 (2002).

${ }^{71}$ J.-C. Tsai and J. P. Gollub, Phys. Rev. E 70, 031303 (2004).

${ }^{72}$ H. S. P. K. Das and B. Jain, Spectrochim. Acta, Part A 60, 2059 (2004).

${ }^{73}$ J. M. Nouri, J. H. Whitelaw, and M. Yianneskis, Laser Anemom. Fluid Mech. 3, 335 (1988).

${ }^{74}$ B. Bailey and M. Yoda, Exp. Fluids 35, 1 (2003).

${ }^{75}$ CRC Handbook of Chemistry and Physics, 85th Ed., edited by D. R. Lide (CRC Press, Boca Raton, 2004).

${ }^{76}$ S. Wu, F. Zeng, H. Wang, W. She, and Z. Cai, J. Appl. Polym. Sci. 89, 2374 (2003)

${ }^{77}$ C. J. Koh, P. Hookham and L. G. Leal, J. Fluid Mech. 266, 1 (1994).

${ }^{78}$ Flat top projector (Coherent).

${ }^{79}$ D. Pine, J. Gollub, J. Brady, and A. Leshansky, Nature (London) 438, 997 (2005).

${ }^{80}$ R. Besseling, L. Isa, E. R. Weeks, and W. C. Poon, Adv. Colloid Interface Sci. 146, 1 (2009).

${ }^{81}$ J. S. Guasto, A. S. Ross, and J. P. Gollub, Phys. Rev. E 81, 061401 (2010).

${ }^{82}$ J. Russ, The Image Processing Handbook (CRC Press, Boca Raton, 2011).

${ }^{83} \mathrm{G}$. Lohmann, Volumetric Image Analysis (Wiley-Teubner, Chichester, 1998).

${ }^{84}$ Experimental and Computational Techniques in Soft Condensed Matter Physics, edited by J. Olafsen (Cambridge University Press, Cambridge, 2010).

${ }^{85}$ J. C. Crocker and D. G. Grier, J. Colloid Interface Sci. 179, 298 (1996).

${ }^{86}$ A. D. Dinsmore, E. R. Weeks, V. Prasad, A. C. Levitt, and D. A. Weitz, Appl. Opt. 40, 4152 (2001).

${ }^{87}$ M. Clusel, E. I. Corwin, A. Siemens, and J. Brujic, Nature (London) 460, 611 (2009).

${ }^{88}$ F. Fahrbach, P. Simon, and A. Rohrbach, Nat. Photonics 4, 780 (2010).

${ }^{89}$ G. Bellani, Ph.D. dissertation, Royal Institute of Technology, KTH Mechanics, Stockholm, 2011.

${ }^{90}$ N. Menon and D. Durian, Science 275, 1920 (1997).

${ }^{91}$ D. Lesniewska and D. Muir Wood, J. Eng. Mech. 135, 1038 (2009).

${ }^{92}$ M. Mathlouthi and P. Reiser, Sucrose: Properties and Applications (Chapman \& Hall, Glasgow, 1995)

${ }^{93}$ J. A. Dijksman, Ph.D. dissertation, Leiden University, Leiden, 2009.

${ }^{94} \mathrm{See}$ http://www.dow.com/surfactants/ for data sheets with a.o. rheological properties of Triton X-100.

${ }^{95}$ I. E. Zarraga and J. D. T. Leighton, Phys. Fluids 14, 2194 (2002).

${ }^{96}$ V. Breedveld, D. van den Ende, A. Tripathi, and A. Acrivos, J. Fluid Mech. 375, 297 (1998).

${ }^{97}$ M. Liva, U.S. patent 4,587,042 (1986); T. Tanaka, U.S. patent 4,789,490 (1988); T. Tanaka, U.S. patent 4,832,855 (1989); I. Motoyama, U.S. patent 6,221,281 (2001).

${ }^{98}$ M. Lyon and L. Leal, J. Fluid Mech. 363, 25 (1998).

${ }^{99}$ B. Bunker, G. Arnold, E. Beauchamp, and D. Day, J. Non-Cryst. Solids 58, 295 (1983).

${ }^{100} \mathrm{See}$ http://www.atsdr.cdc.gov/toxprofiles/tp67-c1-b.pdf for toxicological information about 1-Methylnaphthalene.

${ }^{101}$ E. R. Weeks, J. C. Crocker, A. C. Levitt, A. Schofield, and D. A. Weitz, Science 287, 627 (2000).

${ }^{102} \mathrm{H}$. Capart, private communication (2011).

${ }^{103}$ E. Hecht, Optics (Addison Wesley, San Francisco, 2002).

${ }^{104}$ F. L. Pedrotti and L. S. Pedrotti, Introduction to Optics (Prentice-Hall, London, 1993). 